\title{
Editorial
}

\section{The haemodynamic response to intubation: a perspective}

Tracheal intubation is a noxious stimulus, tending to provoke a marked sympathetic response, manifest as tachycardia and hypertension, which is potentially deleterious in some patients. Various agents effectively attenuate this response, including anaesthetics, analgesics, adrenergic blocking agents, and vasodilators. This has been a fertile area for clinical investigation, spawning numerous studies of the various techniques which might be expected to modify the haemodynamic response to intubation when compared with an unsupplemented sleep dose of thiopentone. Although these investigations invariably document changes in process variables such as heart rate and blood pressure, during the brief periinduction period, they almost never provide information about outcome. Therefore, the influence of these interventions on the incidence of perioperative cardiovascular morbidity and mortality remains unknown.

In this issue of the Joumal, Splinter and Cervenko examine the influence of various doses and combinations of intravenous lidocaine and fentanyl, given prior to induction with thiopentone and succinylcholine, on the haemodynamic response to intubation in the elderly. ${ }^{1}$ Not suprisingly, each agent independently attenuated the response to intubation, and caused minor side-effects. Regimens which effectively blunted both the heart rate and blood pressure response were subsequently associated with mild bradycardia and/or hypotension. The authors conclude that "both lidocaine and low-dose fentanyl are recommended adjuncts to induction of anaesthesia with thiopentone in geriatric patients." However, no outcome data are provided to support this recommendation. Since evidence of myocardial ischaemia was not systematically sought, we cannot decide which regimen would most effectively prevent ischaemia. Therefore, the benefits of the techniques described remain presumptive. Can we assume that prevention of the haemodynamic response to intubation will alter outcome? Do we need, as suggested by Splinter and Cervenko, further investigation ... "to

Department of Anesthesia, St. Boniface General Hospital, Winnipeg. Manitoba. examine the effects of other pharmacological approaches at controlling haemodynamic variables during the induction of anaesthesia in geriatric patients"?

Some patients unquestionably require careful haemodynamic control during induction of anaesthesia and intubation of the trachea. Even a transient hyperdynamic response may cause serious complications in patients with symptomatic aortic aneurysm, recent myocardial infarction, cerebral aneurysm, or intracranial hypertension. Such patients will never be included in the control group of an outcome study examining the efficacy of haemodynamic control at the time of tracheal intubation! In these difficult situations a knowledgeable, experienced anaesthetist will be able to induce anaesthesia without provoking an unacceptable haemodynamic response during intubation. Precisely how the desired haemodynamic result is attained will depend on the clinical scenario and the anaesthetist's preference. The method may be less important than the result.

Known or suspected ischaemic heart disease is by far the most common indication for modifying the haemodynamic response to intubation. The value of haemodynamic control in this population is somewhat controversial. Myocardial ischaemia is a frequent consequence of induction and intubation, especially if tachycardia occurs. ${ }^{2}$ Perioperative myocardial ischaemia has been associated with postoperative myocardial infarction, and a causal relationship has been postulated. ${ }^{2,3}$ Therefore, eliminating ischaemia at the time of intubation might prevent infarction. However, there are reasons to doubt the validity of this syllogism.

Recent studies indicate that frequent, often silent, ischaemic episodes are an everyday occurrence in ambulatory patients with coronary disease. ${ }^{4}$ These ischaemic episodes are generally well tolerated and obviously do not cause infarction. A similar pattem is seen preoperatively in surgical patients with coronary disease. Intraoperative myocardial ischaemia does not occur more frequently than preoperative ischaemia, and may reflect the preoperative ischaemic pattern, rather than the stress of anaesthetic induction and surgery. ${ }^{5}$ Furthermore, a large propor- 
tion of perioperative ischaemic episodes is unrelated to haemodynamic derangements, and is thought to result from decreases in myocardial oxygen supply caused by increasing coronary vascular resistance. ${ }^{2,4,5}$ Haemodynamic control may not eliminate this kind of ischaemia. ${ }^{6}$

The most likely explanation of the association between perioperative ischaemia and infarction is that these are two unrelated manifestations of severe underlying coronary artery disease. Intubation may simply be a stress test which, by causing ischaemia, detects the presence of severe coronary artery disease, and thus predicts adverse outcome. Attenuating the response to intubation might reduce the sensitivity of the stress test without improving outcome.

Myocardial infarction occurs most frequently between the second and fifth postoperative days. Such events seem intuitively unlikely to be related to transient myocardial ischaemia occurring during intubation. The cause of perioperative infarction is unknown, but the terminal event may be coronary thrombosis. ${ }^{7}$ These thromboses may be precipitated by changes in blood coagulability and/or coronary vascular tone which are precipitated by the stress of surgery. If so, then anaesthetic techniques which produce sustained suppression of the neuroendocrine stress response might be associated with improved outcome. Such a program might include preoperative adrenergic blockade, blunting of the neuroendocrine response to intubation and surgery, and effective, continuous control of postoperative pain. Haemodynamic variables may provide us with an on-line monitor of the effectiveness of stress suppression. Recent studies support the hypothesis that controlling perioperative stress improves outcome in high-risk patients. ${ }^{8.9}$

In conclusion, modification of the haemodynamic response to intubation is a laudable objective and is clearly indicated in a small subgroup of patients in whom a single hyperdynamic episode may cause a catastrophe. However, suppression of the haemodynamic response to intubation is, by itself, unlikely to influence outcome in patients with stable coronary artery disease. However, extension of the principle of haemodynamic control to the entire perioperative period may prove beneficial. Continuing to focus on tracheal intubation may cause tunnel vision. We should expand our horizon, and attempt to control haemodynamic variables for 72 hours perioperatively, rather than for two minutes following intubation. The probability of benefit may be proportional to the duration of haemodynamic control.

\section{La réponse hémo- dynamique à l'intu- bation: un perspective}

L'intubation de la trachée amène souvent une forte réponse sympathique avec tachycardie et hypertension mal tolérés par certains patients. Plusieurs anesthésiques, analgésiques, bloquants adrénergiques et vasodilatateurs peuvent atténuer ce réflexe et on les a mis à profit dans le cadre de nombreux protocoles de recherche comparant diverses combinaisons au thiopental employé seul. Invariablement, ces études ont démontré qu'il y avait moyen de réduire les variations du pouls et de la pression artérielle pendant la période péri-induction sans toutefois fournir d'information sur le sort ultime des patients. On ne sait donc trop ce que ces techniques changent en bout de ligne sur la morbidité cardiovasculaire péri-opératoire.

On retrouvera dans ce numéro du Journal les observations des docteurs Splinter et Chervenko quant à l'effet de diverses doses et combinaisons de lidocaïne et de fentanyl injectées avant le thiopental et la succinylcholine sur la réponse hếmodynamique à l'intubation de patients âgés. ${ }^{1}$ Ainsi, chacun des deux agents atténuait l'impact de l'intubation au prix d'effets secondaires mineurs. Ces agents, capables de réduire la tachycardic et l'hypertension à l'intubation, ont plus tard amené de la bradycardie et/ou de l'hypotension. Les auteurs concluent que: "le fentanyl à faible dose et/ou la lidocaïne devraient être associés au thiopental lors de l'induction de l'anesthésie chez les patients âgés".

Malheureusement, nous ne savons pas ce qui est finalement arrivé à leurs patients et comme il n'y avait pas de mesure de l'ischémie myocardique, on ne sait trop comment elle pouvait le mieux être prévenue. Alors, est-il sage de présumer que la prévention des fluctuations hémodynamiques lors de l'intubation va changer le sort des patients? Avons nous vraiment besoin d'autres études qui établiront de nouvelles techniques de contrôle des variables hémodynamiques lors de l'induction de l'anesthésie chez le patient âgé comme le soutiennent Splinter et Chervenko?

Certains patients requièrent hors de tout doute un contrôle hémodynamique serré lors de l'induction anesthésique et de l'intubation de la trachée. Les victimes récentes d'un infarctus du myocarde, les porteurs d'anévrysme cérébral ou d'anévrysme disséquant de l'aorte ou ceux dont la pression intracrânienne est élevée sont à risque advenant un épisode d'hyperdynamie. Il est 
cependant fort peu probable que ces demiers fassent jamais partie du groupe contrôle d'une étude visant à démontrer l'effet de la réponse hémodynamique à l'intubation sur la morbidité! En évaluant la situation et en se fiant à son expérience, l'anesthésiste chevronné aux prises avec un cas semblable saura manoeuvrer pour éviter une catastrophe hémodynamique lors de l'intubation; la méthode exacte employée importera alors moins que le résultat.

La présence d'une maladie coronarienne est la raison invoqué le plus souvent pour chercher à atténuer la réponse hémodynamique à l'intubation. Pourtant, la valeur du contrôle hémodynamique chez les coronariens reste à démontrer. En fait, l'induction et l'intubation amènent souvent de l'ischémie myocardique surtout s'il y a de la tachycardie. ${ }^{2}$ On a par ailleurs associé l'ischémie myocardique peri-opératoire avec les infarctus du myocarde survenant en post-opératoire, postulant même une relation causale entre les deux. ${ }^{2,3}$ Ainsi, en évitant l'ischémie lors de l'intubation, on pourait peut-être prévenir certains infarctus. On peut mettre en doute ce raisonnement un peu court.

De nouvelles études viennent de démontrer que des épisodes d'ischémie souvent silencieux surviennent quotidiennement chez les coronariens actifs et qu'ils sont habituellement bien tolérés et sans conséquence. ${ }^{4}$ En pré-opératoire, les coronariens montrent cette même propension à l'ischémie et cette dernière se manifeste aussi en période opératoire et ce à la même fréquence, reflet possible de la rythmicité intrinsèque de la maladie plutốt que du stress de l'induction anesthésique et de la chirurgie. ${ }^{5}$ De plus, de nombreux épisodes ischémiques peri-opératoires surviennent en l'absence de tout changement hémodynamique et pourraient résulter d'une baisse de l'apport myocardique en oxygène causée par une augmentation de la résistance des coronaires. ${ }^{2,4,5}$ Ce type d'ischémie est donc indépendant du contrôle de l'hémodynamie. $^{6}$

L'association entre l'ischémie périopératoire et l'infaretus est probablement due au fait que tous deux reflètent indépendamment la sévérité de la maladie sousjacente. L'intubation n'est peut-être qu'une épreuve d'effort qui causant de l'ischémie, mets en évidence la gravité de la maladie coronarienne et prédit du même coup le risque d'infarctus. Si on modifie la réponse à l'intubation, on ne "verra" peut-être pas la sévérité de l'atteinte coronaire mais le résultat final sera le même.

D'ailleurs, les infarctus du myocarde surviennent habituellement entre le deuxième et le cinquième jour post-opératoire et il serait imprudent d'en chercher la cause dans un épisode ischémique limité lors de l'intubation. Les causes exactes de ces infarctus ne sont pas claires mais éventuellement, il y a thrombose de la coronaire. ${ }^{7}$ De l'hypercaogulabilité sanguine et/ou une augmentation du tonus des coronaires suite au stress chirurgical pourraient expliquer cette propension aux thromboses. Il y aurait alors avantage à utiliser une technique anesthésique supprimant de façon prolongée la réponse neuroendocrinienne au stress, non seulement lors de l'intubation et de la chirurgie mais aussi en préopératoire avec des bloquants adrénergiques et en post-opératoire avec une analgésie efficace et constante. La mesure des variables hémodynamiques pourrait alors nous informer sur la qualité de la suppression du stress. Deux nouvelles études viennent d'étayer l'hypothèse que le contrôle du stress péri-opératoire réduit la morbidité chez les patients à risque. ${ }^{8,9}$

Donc, l'atténuation de la réponse hémodynamique à l'intubation est un objectif louable et nécessaire chez les quelques patients pour qui un simple épisode hyperdynamique équivaudrait à une catastrophe. Cependant, cela seul ne changera probablement pas le sort des coronariens stables. Toutefois, il y aurait peut-être avantage à étendre le principe du contrôle hémodynamique à toute la période péri-opératoire en élargissant nos horizons au delà de l'intubation jusqu'au troisième jour post-opératoire. Les avantages pressentis du contrôle hémodynamique pourraient être proportionnels à sa durée.

\section{References}

1 Splinter WM, Cenvenko $F$. Haemodynamic responses to laryngoscopy and tracheal intubation in geriatric paticnts: effects of fentanyl, lidocaine and thiopentone. Can J Anaesth 1989; 36: 370-6.

2 Slogoff $S$, Keats $A S$. Does perioperative myocardial ischemia lead to postoperative myocardial infarction? Anesthesiology 1985; 62: 107-14.

3 Lowenstein E. Perianesthetic ischemic episodes cause myocardial infarction in humans - a hypothesis confirmed. Anesthesiology 1985; 62: 103-6.

4 Deanfield JE, Selwyn AP, Chierchia S et al. Myocardial ischaemia during daily life in patients with stable angina: its relation to symptoms and heart rate changes. Lancet 1983; 2: 753-8.

5 Knight AA, Hollenberg $M$, London $M J$ et al. Perioperative myocardial ischemia: importance of the preoperative ischemic pattem. Anesthesiology 1988; 68: 681-8.

6 Slogoff S, Keats AS. Randomized trial of primary anesthetic agents on outcome of coronary artery bypass operations. Anesthesiology 1989; 70: 179-88.

7 Davies MJ, Woolf N, Robertson WB. Pathology of acute myocardial infarction with particular reference to occlusive coronary thrombi. Br Heart J 1976; 38: 659.

8 Yeager MP, Glass DD, Neff RK, Brinck-Johnsen T. Epidural anesthesia and analgesia in high risk surgical patients. Anesthesiology 1987; 66: 729-36.

9 Roizen MF, Lampe GH, Benefiel DJ et al. Is increased operative stress associated with worse outcome? Anesthesiology 1987; 67: A1. 